
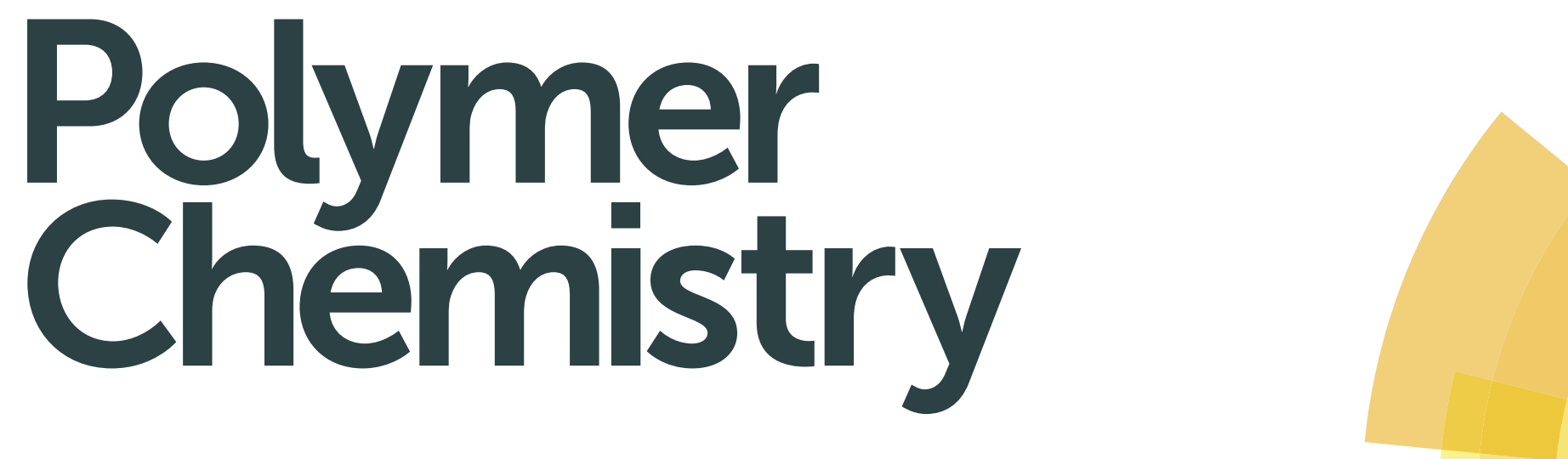

www.rsc.org/polymers

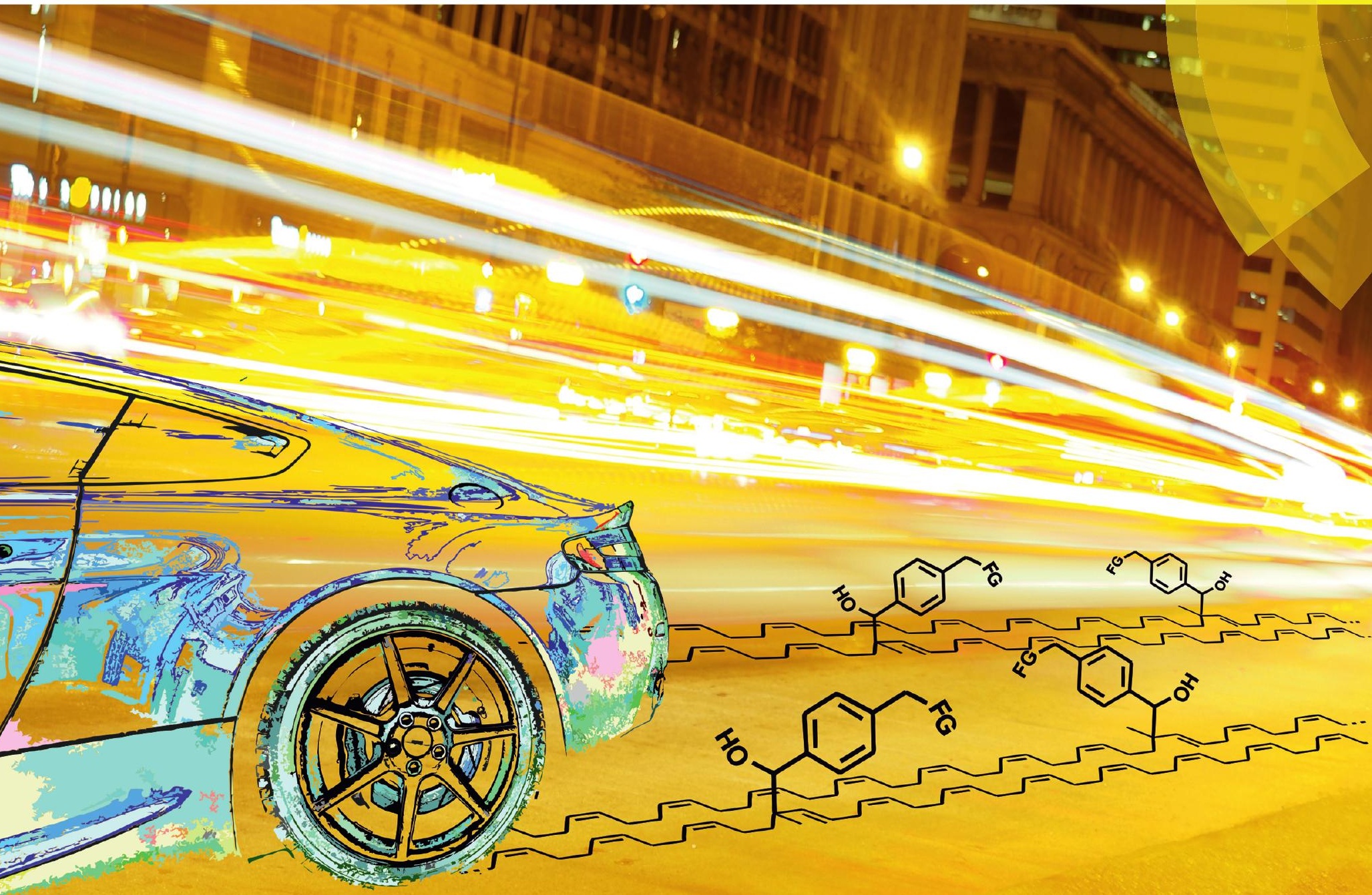

ISSN 1759-9954

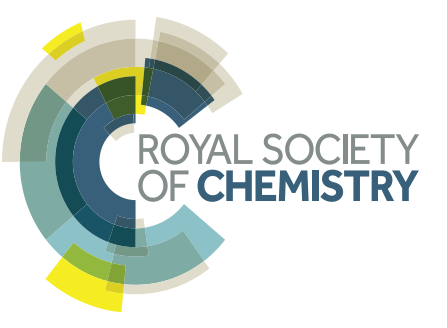




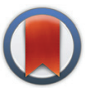

CrossMark $\leftarrow$ click for updates

Cite this: Polym. Chem., 2016, 7, 7195

Received 9th August 2016,

Accepted 17th September 2016

DOI: $10.1039 /$ c6py01388k

www.rsc.org/polymers

\title{
Allylboration as a versatile tool for the in situ post-polymerization functionalization of 1,4-cis-poly(butadiene) $\uparrow$
}

\author{
Hannes Leicht, Steffen Huber, Inigo Göttker-Schnetmann and Stefan Mecking*
}

\begin{abstract}
Allylboration is a versatile tool for the post-polymerization functionalization of poly(butadiene-co-[4,4,5,5-tetramethyl-2-(3methyl-1,3-butadienyl)-1,3,2-dioxaborolane]). Polar functionalized aldehydes $\mathrm{HC}(\mathrm{O}) \mathrm{C}_{6} \mathrm{H}_{4}\left(\mathrm{CH}_{2}\right) \mathrm{R}\left(\mathrm{R}=\mathrm{Br}, \mathrm{NR}_{2}, \mathrm{PPh}_{3}, \mathrm{P}(\mathrm{O})(\mathrm{OEt})_{2}\right)$ react readily with the allyl boronic acid ester groups in the copolymer without interfering with the reactive double bonds in the polymer backbone. This provides access to stereoregular poly(butadiene) functionalized with a broad range of polar groups. Functionalization proceeds under polymerization conditions and therefore does not require a prior polymer work-up.
\end{abstract}

The synthesis of copolymers based on polar and nonpolar olefins via insertion polymerization is a challenging goal of polymer chemistry. Fundamental progress has been made recently in the polymerization of ethylene with polar vinyl monomers. ${ }^{1-4}$ The functionalization of poly(dienes) via direct copolymerization with polar monomers, however, is almost exclusively accomplished by free-radical or anionic methods. ${ }^{5-8}$ Polar functionalized poly(dienes) thus obtained can possess superior interactions with typical filler materials in tire applications such as silica or carbon black. ${ }^{9,10}$ However, the lack of microstructure control in these free-radical or anionic polymerizations is a major drawback because the properties of the poly(dienes), and hence their applicability, are strongly dependent on their polymer microstructure. Although progress has been made very recently towards copolymerizing 1,3-butadiene (BD) and isoprene (IP) with a broad variety of polar functionalized dienes to stereoregular copolymers, there are still functionalities that withdraw themselves from direct copolymerization. ${ }^{9,11}$ Post-polymerization functionalization is widely applied in poly(diene) chemistry. Vulcanization in the rubber industry is a prominent example. The reactivity of sulfur compounds with double bonds was also applied in the functionalization of 1,2-poly(butadiene) and poly(isoprene-co-

Chair of Chemical Materials Science, Department of Chemistry, University of Konstanz, 78464 Konstanz, Germany. E-mail: stefan.mecking@uni-konstanz.de $\dagger$ Electronic supplementary information (ESI) available: Selected NMR-spectra and synthetic procedures. See DOI: 10.1039/c6py01388k 3-methylenehepta-1,6-diene) via the thiol-ene reaction. ${ }^{10,12-14}$ Although the functionalizations were successful, they still require additional activation, namely UV-irradiation and/or the addition of radical initiators. However, this approach cannot be utilized for high 1,4-cis poly(butadiene) (PBD) as cross-linking occurs under the functionalization conditions. ${ }^{13}$ A mild method for a versatile post-polymerization functionalization of 1,4-cis-poly(butadiene) with a reactivity approach orthogonal to the backbone's double bonds is highly desirable.

For this purpose we explored the allylboration reaction of allyl boronic acid ester groups (Scheme 1) in poly(butadiene$c o$-[4,4,5,5-tetramethyl-2-(3-methyl-1,3-butadienyl)-1,3,2-dioxaborolane]) for post-polymerization reactions to introduce functional groups. High 1,4-cis-poly(butadiene-co-[4,4,5,5-tetramethyl-2-(3-methyl-1,3-butadienyl)-1,3,2-dioxaborolane]) was synthesized straightforwardly via direct insertion copolymerization of $\mathrm{BD}$ and 4,4,5,5-tetramethyl-2-(3-methyl-1,3butadienyl)-1,3,2-dioxaborolane catalyzed by the cationic nickel complex [(allyl)Ni(mesitylene)](BAr $\left.{ }_{4}^{\mathrm{F}}\right)$ Ni-1. ${ }^{11} \quad 4,4,5,5-$ Tetramethyl-2-(3-methyl-1,3-butadienyl)-1,3,2-dioxaborolane was chosen as a comonomer, because it is easily accessible through hydroboration of 2-methylbut-1-en-3-yne in excellent yields and high purity. Additionally, it readily generates the desired allyl boronic acid ester group in the polymer backbone upon insertion (Scheme 1).

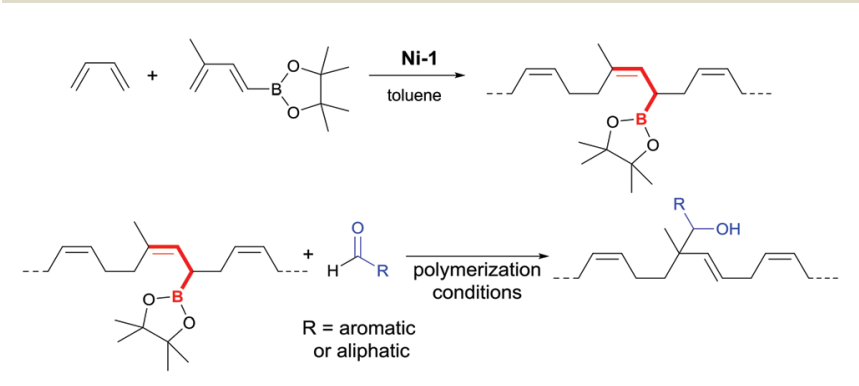

Scheme 1 Formation of an allyl boronic acid ester group in the copolymer employed and conversion in an allylboration reaction. 
Initial experiments with an allylboronic acid pinacol ester as a small molecule model compound were designed to investigate the behavior of the reaction under conditions comparable to those of the desired application. All model reactions with the allylboronic acid pinacol ester and different aldehydes (pentanal, benzaldehyde, $p$ - $\mathrm{NO}_{2}$-benzaldehyde, $p$-dimethylaminobenzaldehyde, and 4-(1-pyrrolidinyl)benzaldehyde) showed the allylboration reaction to be a potentially robust and easy method for the introduction of functional groups into the backbone of poly(dienes). The expected products were formed in good (e.g. 73\% with $p$-dimethylaminobenzaldehyde) to high yields (e.g. $89 \%$ with $p$ - $\mathrm{NO}_{2}$-benzaldehyde) as observed by means of ${ }^{1} \mathrm{H}$ NMR.

To assess the general reactivity of aldehydes towards the allyl boronic acid ester groups in the polymer backbone, $500 \mathrm{mg}$ copolymer were reacted with 10 equiv. benzaldehyde $\left(60{ }^{\circ} \mathrm{C}, 2-3\right.$ days in $5 \mathrm{~mL}$ toluene). The excess of benzaldehyde was removed subsequent to the reaction by precipitation of the polymer in methanol. After drying under reduced pressure, the polymer was characterized comprehensively by NMR spectroscopy.

The full conversion of the allylboronic acid pinacol ester groups in the copolymer to the desired secondary alcohol is evident by comparing key signals in the copolymer before and after the allylboration reaction (Fig. 1). While the signals for the vinylic $\mathrm{CH}_{3}$ group 1, the olefinic proton 2, and the $\mathrm{CH}_{3}$ groups of the pinacol ester 3 disappear, a set of new key resonances can be found in the product. These key resonances include the $\mathrm{OH}$-substituted $\mathrm{CH}$ group 4 with a distinctive shift in ${ }^{1} \mathrm{H}$ (4.38 ppm) as well as ${ }^{13} \mathrm{C}$ NMR (80.8 ppm). In addition, a signal for the newly formed biallylic $\mathrm{CH}_{2}$ group 5 resonates at $\delta=2.79 \mathrm{ppm}\left({ }^{13} \mathrm{C}: 30.8 \mathrm{ppm}\right)$, the peak of the $\mathrm{CH}_{3}$ group 1 shifts from $1.69 \mathrm{ppm}$ to $1.04 \mathrm{ppm}$, and the signals of the aromatic protons of the aryl ring 7 appear between 7.15 and $7.32 \mathrm{ppm}$. These observations and the chemical shift of the quaternary carbon atom $8(44.5 \mathrm{ppm})$ prove the nucleophilic substitution to proceed highly selectively in an $\mathrm{S}_{\mathrm{N}} 2^{\prime}$ fashion. Signals indicating the formation of the product formed in the substitution following an $\mathrm{S}_{\mathrm{N}} 2$ mechanism or other side-reactions were not observed.

To enlarge the scope of this functionalization approach, further different aldehydes were reacted with the copolymer: the full conversion of the allylboronic acid pinacol ester groups of the copolymer with pentanal under otherwise identical conditions showed that the reactivity is not limited to aromatic aldehydes and also that alkyl aldehydes are suitable reagents for functionalization of diene copolymers via an allylboration reaction. Quantitative conversions are also observed when commercially available, N-functionalized, aromatic aldehydes are used. Both, 4-(1-pyrrolidinyl)benzaldehyde and $p$-dimethylaminobenzaldehyde were individually reacted for two days with the copolymer under otherwise identical conditions as the reaction with benzaldehyde. NMR analyses after repeated precipitation of the polymers showed in both cases additional signals compared to the polymer functionalized with benzaldehyde: The polymer functionalized with 4-(1pyrrolidinyl)benzaldehyde exhibits both ${ }^{1} \mathrm{H}$ and ${ }^{13} \mathrm{C}$ signals for the pyrrolidine moiety at $\delta=3.28 \mathrm{ppm}(47.7 \mathrm{ppm})$ and $2.00 \mathrm{ppm}(25.6 \mathrm{ppm})$. In the case of $p$-dimethylaminobenzaldehyde, both methyl groups resonate at $2.94 \mathrm{ppm}$ as a key signal in the proton NMR spectrum.

The aforementioned studies were all conducted with a separately synthesized copolymer. However, functionalization is desirable without an additional step (i.e. work-up of the polymer) directly after the copolymerization of butadiene with 4,4,5,5-tetramethyl-2-(3-methyl-1,3-butadienyl)-1,3,2-dioxaborolane. For this purpose, we ran two copolymerizations under standard polymerization conditions. ${ }^{15}$ At the end of both polymerizations, benzaldehyde (42 equiv. to comonomer) or 4-(1-pyrrolidinyl)benzaldehyde (10 equiv. to comonomer) was added. Both reaction mixtures were then stirred at $50{ }^{\circ} \mathrm{C}$ and the conversion was followed by ${ }^{1} \mathrm{H}$ NMR taking aliquots. The reaction with benzaldehyde showed a degree of functionalization of $c a .50 \%$ after $48 \mathrm{~min}$ and complete functionalization after two hours. The reaction using 4-(1pyrrolidinyl)benzaldehyde, however, proceeded significantly
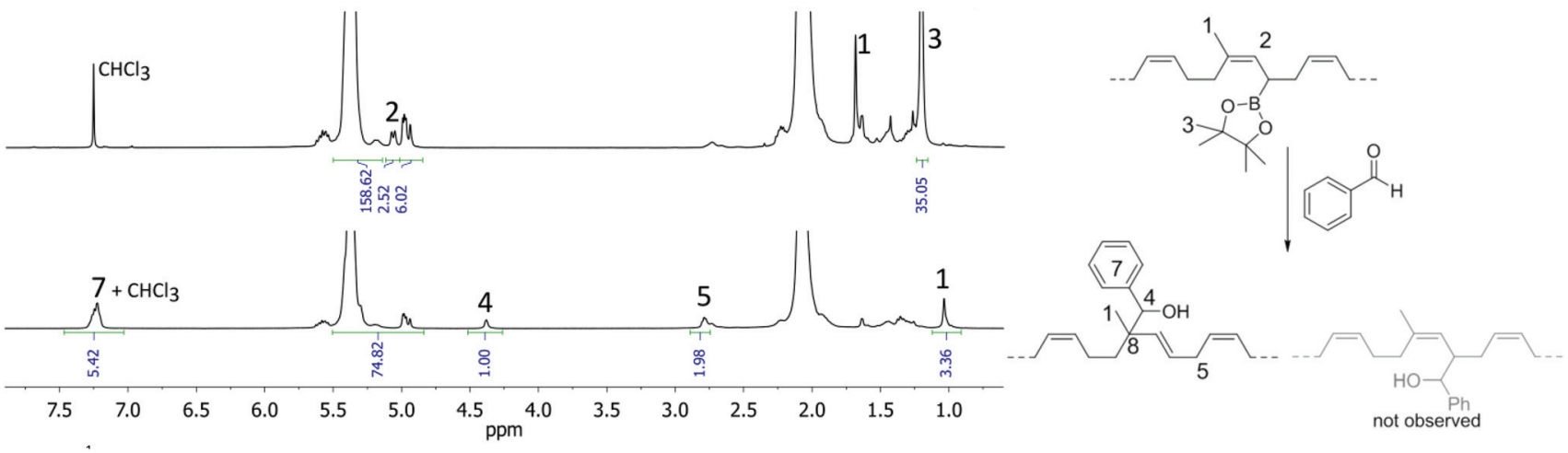

Fig. $1{ }^{1} \mathrm{H}$ NMR spectra of the copolymer before and after the allylboration reaction with benzaldehyde showing the selective formation of the product structure expected for an $\mathrm{S}_{\mathrm{N}} 2$ ' substitution mechanism. 
slower. After $2.5 \mathrm{~h}$ only $25 \%$ of the allylboronic acid ester groups in the copolymer were converted. A conversion of $50 \%$ was reached after $28 \mathrm{~h}$ and full functionalization was reached after two days. This difference in the reaction rates is possibly due to the different amounts of aldehyde compared to that of the comonomer, or due to a deactivating influence of the amine group on the para-position to the aldehyde moiety.

This outcome prompted us to engage in the synthesis of aromatic aldehydes with a methylene group as a spacer between the aromatic ring and the functional group.

Additionally, this approach should generate a platform to synthesize aromatic aldehydes with different functional groups.

Syntheses of differently functionalized aromatic aldehydes were accomplished via two different routes (Scheme 2). Route (A) encompasses the functionalization of 1-bromo-4-(bromomethyl)benzene with a nucleophile (e.g. pyrrolidine) followed by conversion to the desired aromatic aldehyde by reaction with $n$-BuLi and DMF followed by an acidic aqueous work-up. Both steps give the desired product in high yields and purity, making purification steps like distillation or column chromatography unnecessary. However, not all functional groups that can be introduced this way are stable towards the conditions in the second step. Therefore, we also used a second route to synthesize functionalized aromatic aldehydes. Route (B) starts with the synthesis of 4-(bromomethyl)benzaldehyde which can be directly reacted with a nucleophile (e.g. $\mathrm{PPh}_{3}$ ). If the nucleophile is reactive towards the aldehyde group (e.g. $\left.\mathrm{NaP}(\mathrm{O})(\mathrm{OEt})_{2}\right)$, the application of well-known protecting group chemistry enables the successful synthesis of the desired product. Except for the first step, the synthesis of 4-(bromomethyl)benzaldehyde, no further purification was necessary for the obtained products.

To gain more information about the reaction's actual progress over time influenced by the para-substituents, we followed the reaction of the copolymer with benzaldehyde, 4-(1-pyrrolidinyl)benzaldehyde, and 4-(pyrrolidinylmethyl) benzaldehyde by ${ }^{1} \mathrm{H}$ NMR (12 equiv. of aldehyde to comono-
Table 1 Different aldehydes used in the allylboration reaction, showing the influences of substitution pattern and equivalents used (compared to functional groups present in the copolymer)

\begin{tabular}{lll} 
Entry & Aldehyde & $\begin{array}{l}\text { Conversion of the polymers' } \\
\text { Equiv. }\end{array}$ \\
\hline
\end{tabular}

Reaction conditions: $65 \mathrm{mg}$ polymer, $0.6 \mathrm{~mL} \mathrm{CDCl}_{3}, 60{ }^{\circ} \mathrm{C}$. ${ }^{a}$ Equivalents of aldehyde compared to functional groups present in the polymer. ${ }^{b}$ Conversion of the functional groups in the polymer, determined from ${ }^{1} \mathrm{H}$ NMR spectra. ${ }^{c}$ Additional 0.5 equiv. of aldehyde were added after $22.5 \mathrm{~h}$, full conversion was observed after additional $2.3 \mathrm{~h}$.

mer units, $60{ }^{\circ} \mathrm{C}$, Table 1 entries $\left.1-3\right)$. The reactivity differences of the compared aldehydes are significant: The conversion with benzaldehyde reached $60 \%$ after $10 \mathrm{~min}$ and full conversion after 75 min (Table 1, entry 1). In comparison, the reaction with 4-(1-pyrrolidinyl)benzaldehyde is much slower as $38 \%$ conversion is reached after $235 \mathrm{~min}$ and full conversion requires heating overnight (Table 1 , entry 2 ).

The reaction with 4 -(pyrrolidinylmethyl)benzaldehyde is again significantly faster and proceeds with rates comparable to those observed for the allylboration reaction using benzaldehyde (full conversion after 95 min, Table 1, entry 3).

The high reactivities of the synthesized functionalized aromatic aldehydes and the clean and selective formation of the
A)
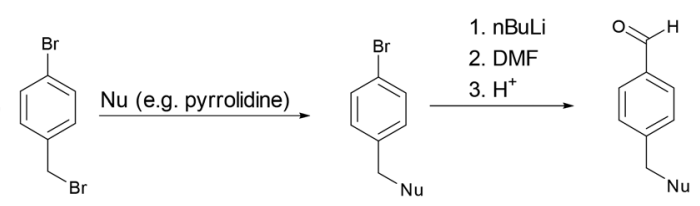

$\mathrm{Nu}\left(\right.$ e.g. $\mathrm{PPh}_{3}$ )

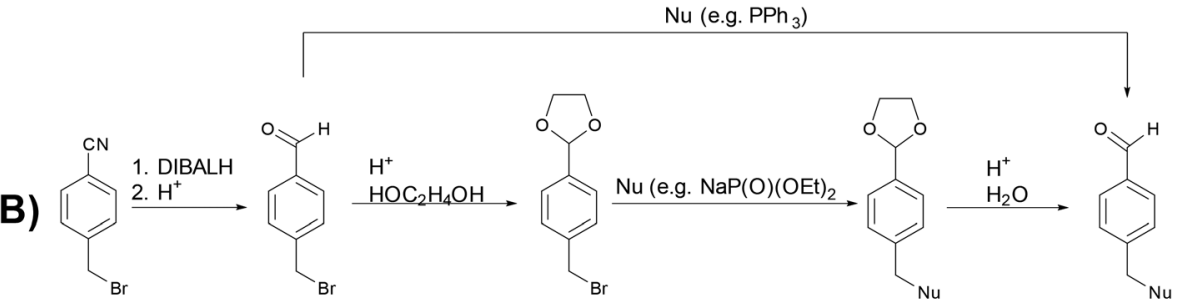

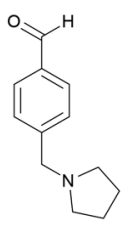

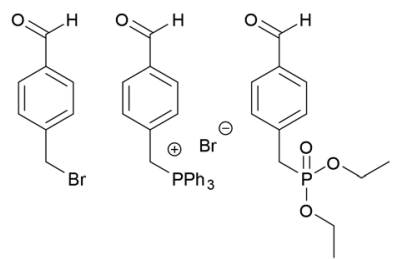

Scheme 2 Syntheses of differently substituted aromatic aldehydes with a $\mathrm{CH}_{2}$-linker. 


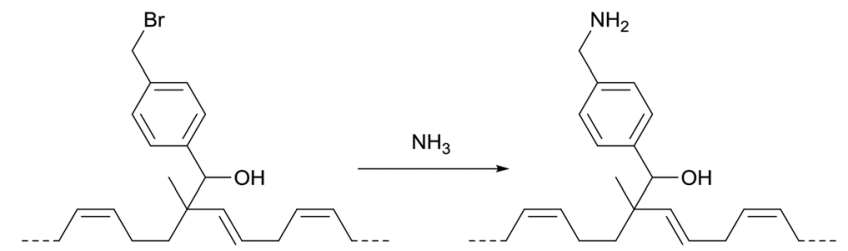

Scheme 3 Generation of $-\mathrm{NH}_{2}$ groups by reaction of a product of allylboration with ammonia.

desired target structure allows the use of equimolar or even substoichiometric amounts of aldehyde (Table 1, entries 3-6). Although longer reaction times are required for equimolar reactions, a waste of reagents is avoided. Allylboration reactions with all synthesized aromatic aldehydes were successfully performed and the obtained functionalized polymers were scrutinized by NMR-spectroscopy to prove the complete functionalization of 1,4-cis-poly(butadiene) (for NMR spectra and assignments $c f$. the ESI $\dagger$ ).

The polymer obtained with 4-(bromomethyl)benzaldehyde (entry 4) can be functionalized further by virtue of the electrophilic nature of the bromobenzyl-moiety. Notably, this allowed the introduction of primary amine groups by reaction with ammonia (Scheme 3; for details see the ESI $\dagger$ ).

In conclusion, we have shown that the allylboration reaction is a versatile and robust tool for the post-polymerization functionalization of 1,4-cis-poly(butadiene). Allyl boronic acid ester groups in poly(butadiene-co-[4,4,5,5-tetramethyl-2-(3methyl-1,3-butadienyl)-1,3,2-dioxaborolane]) react readily with para-functionalized aromatic aldehydes to give access to functionalized high 1,4-cis-poly(butadienes) that are not accessible via a direct copolymerization approach. The allylboration reaction can be performed directly after polymerization without the need for a prior work-up of the polymer and proceeds quantitatively in an $\mathrm{S}_{\mathrm{N}} 2^{\prime}$ fashion without the occurrence of side-reactions.

\section{Acknowledgements}

We thank Karen Burke, Maggie Flook, and Stephan Rodewald for fruitful discussions. The Goodyear Tire \& Rubber Company is gratefully acknowledged for financial support.

\section{Notes and references}

1 E. Y. X. Chen, Chem. Rev., 2009, 109, 5157-5214.

2 E. Drent, R. van Dijk, R. van Ginkel, B. van Oort and R. I. Pugh, Chem. Commun., 2002, 744-745.

3 L. K. Johnson, S. Mecking and M. Brookhart, J. Am. Chem. Soc., 1996, 118, 267-268.

4 A. Nakamura, S. Ito and K. Nozaki, Chem. Rev., 2009, 109, 5215-5244.

5 V. V. Sheares, L. Wu, Y. Li and T. K. Emmick, J. Polym. Sci., Part A: Polym. Chem., 2000, 38, 4070-4080.

6 K. Sunada, K. Takenaka and T. Shiomi, J. Appl. Polym. Sci., 2005, 97, 1545-1552.

7 Y. Yang, J. Lee, M. Cho and V. V. Sheares, Macromolecules, 2006, 39, 8625-8631.

8 Y. Yang and V. V. Sheares, Polymer, 2007, 48, 105-109.

9 C. Yao, N. Liu, S. Long, C. Wu and D. Cui, Polym. Chem., 2016, 7, 1264-1270 and references cited therein.

10 L. Li, S. H. Li and D. M. Cui, Macromolecules, 2016, 49, 1242-1251 and references cited therein.

11 H. Leicht, I. Göttker-Schnetmann and S. Mecking, ACS Macro Lett., 2016, 5, 777-780.

12 R. L. A. David and J. A. Kornfield, Macromolecules, 2008, 41, 1151-1161.

13 J. Justynska, Z. Hordyjewicz and H. Schlaad, Polymer, 2005, 46, 12057-12064.

14 N. Ten Brummelhuis, C. Diehl and H. Schlaad, Macromolecules, 2008, 41, 9946-9947.

$1520 \mu \mathrm{mol} \mathrm{Ni-1,} 20 \mathrm{~mL}$ toluene, 1.05 bar BD, 0.7-0.8 mmol comonomer, $25^{\circ} \mathrm{C}, 30 \mathrm{~min}$. 